\title{
Safety Profile, Pros and Cons of the Submental Flap in Reconstruction of the Orofacial Defects after Malignant Tumor Resection
}

\author{
MOHAMMED HASAN OSMAN, M.D. \\ The Department of Maxillofacial Surgery Unit, Faculty of Medicine, Assiut University, Assiut, Egypt
}

\begin{abstract}
Objective: The main aim of this paper is to assess the safety profile and usefulness of the submental flap as a reconstructive tool for reconstructing orofacial defects after malignant tumor resection.
\end{abstract}

Methods: A total sample of 23 patients suffering from oral cavity cancers during two years were included. All patients underwent surgical excision of the primary tumor and neck dissection with immediate reconstruction of the resultant defect using the submental flap.

Results: Among patients included in the study sample, there were 15 males and 8 females. They were in the age group of 39 to 67 years old. Chin (1) buccal mucosa (5), alveolar margin (4), and tongue (13) were the primary tumor sites. After the operation, the evaluation of functions, swallowing, speaking, and jaw movements were all good in all patients. Advantages include long rotation arc, short operative time, short hospital stay, and excellent healing of the donner site. Disadvantages include the persistence of hair in male patients and recurrences in 5 patients.

Conclusion: The study concluded that one of the best instruments for reconstructing orofacial tissue is the submental flap. It is easily harvested, valid, and locally available flap. However, it is necessary to use the submental flap with adequate care in patients with a positive neck.

Key Words: Neck dissection - Surgical flaps - Submental flap - Oral carcinoma - Reconstruction.

\section{INTRODUCTION}

Oral cavity carcinomas are the $6^{\text {th }}$ most common cancer worldwide and represent nearly $30 \%$ of all head and neck cancers [1]. The standard treatment is surgical excision, with or without regional neck dissection, where the primary tumor is excised with a safety margin of at least $1-1.5 \mathrm{~cm}$ all around [2]. Multiple reconstruction methods have been introduced to reconstruct the orofacial defects after these ablative surgeries, including split-thickness skin grafts, locoregional flaps, and free flaps. Each of these techniques has its advantages and disadvantages [3].

The submental island flap is one of these methods. Since it was introduced by Martin et al., in
1993, it gained great popularity in reconstructing small to medium-sized orofacial defects [4]. Although this flap has several advantages, including an excellent skin color match and a wide arc of rotation, its oncological safety still of great concern [5]. This controversy comes from the fact that the submental vessels arise from the facial vessels at the level I group of the neck lymph nodes, the first-echelon lymphatic drainage basin for oral cavity carcinomas.

Consequently, this article presents our experience of this flap in reconstructing orofacial defects after malignant oral tumor resection and assessing its oncological safety, pros, and cons.

\section{PATIENTS AND METHODS}

\section{Patients:}

A total sample of 23 patients suffering from oral cavity carcinoma was included in this study from March 2016 to March 2018 in the Maxillofacial Unit, Surgery Department, Assiut university hospital, Egypt. This work was performed in accordance with the Declaration of Helsinki and was reviewed and approved by The Medical Ethics Committee of the Faculty of Medicine, Assiut University (IRB approval number: 17300487). All participants provided their written, informed consent to participate in this study.

\section{Selection criteria:}

1- Patients diagnosed with operable orofacial carcinoma by pathologic examination.

2- Patients had no history of surgical excision or chemoradiotherapy treatment for the oral maxillofacial region.

3- Patients agree to participate in this study.

4- Patients fit for surgery and general anesthesia.

All patients underwent a preoperative clinical examination with neck ultrasound and CT scan or magnetic resonance imaging of the cervicofacial 
area. Patients' age, sex, histopathological features, and the stage of their tumors were all analyzed.

\section{Surgical technique:}

Following the oncologic principles, the primary tumor was resected with a $1-1.5 \mathrm{~cm}$ safety margin all around with neck dissection, and the resulting defect was reconstructed immediately using the submental artery flap.

Some surgical tricks had to be followed in all the cases of this study during flap harvesting (Figs. 1,2,3):

- To determine the maximal flap width, we applied a pinch test for the facilitation of primary closure.

- Neck dissection was started and completed before flap harvesting.

- Preservation of the facial vessels was a crucial part of the surgery.
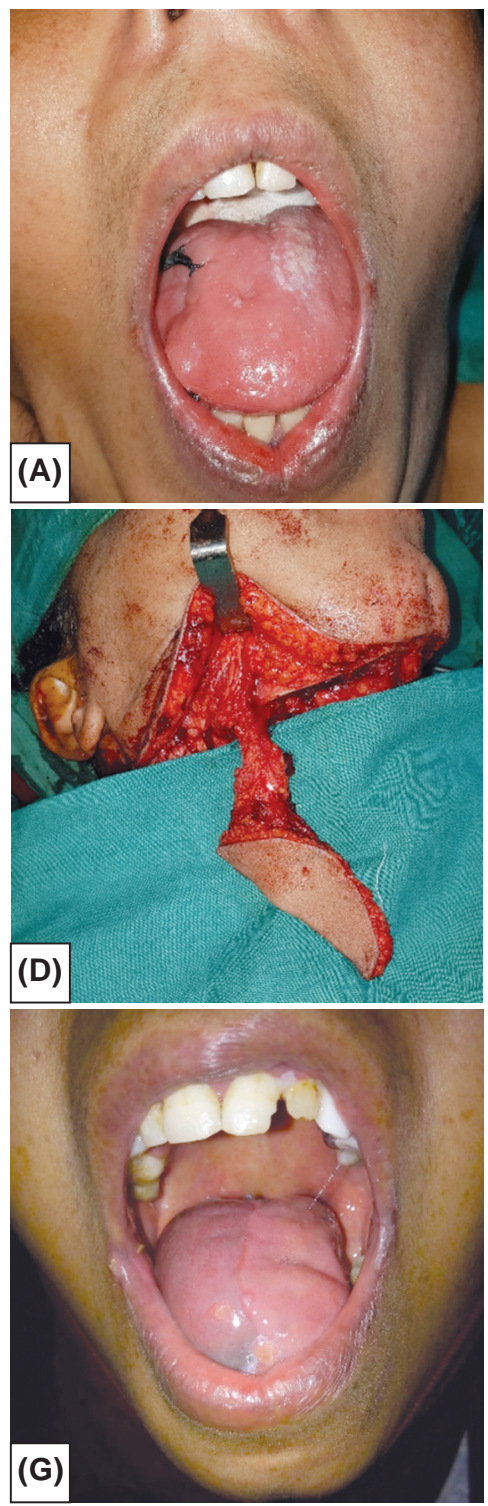

- The marginal mandibular nerve was identified and preserved after the elevation of the subplatysmal flap. The submental artery was identified after exposure of the facial artery and dissected away from the submandibular gland. Level Ib lymph nodes were carefully excised, and the submental triangle (level Ia) lymph nodes anterior to the anterior digastric were excised separately.

- Flap elevation began from the contralateral side of the pedicle in the subplatysmal plane.

- We included the anterior belly of the digastric muscle in all flaps to improve the flap's viability.

- Further, we included a part of the mylohyoid muscle to protect perforating vessels and enhance venous drainage.

- We rooted the flap lateral to the mandible when the primary tumor included the buccal mucosa, alveolar margin, or chin, and we rooted the flap medial to the mandible when the primary tumor included the tongue or oral floor.
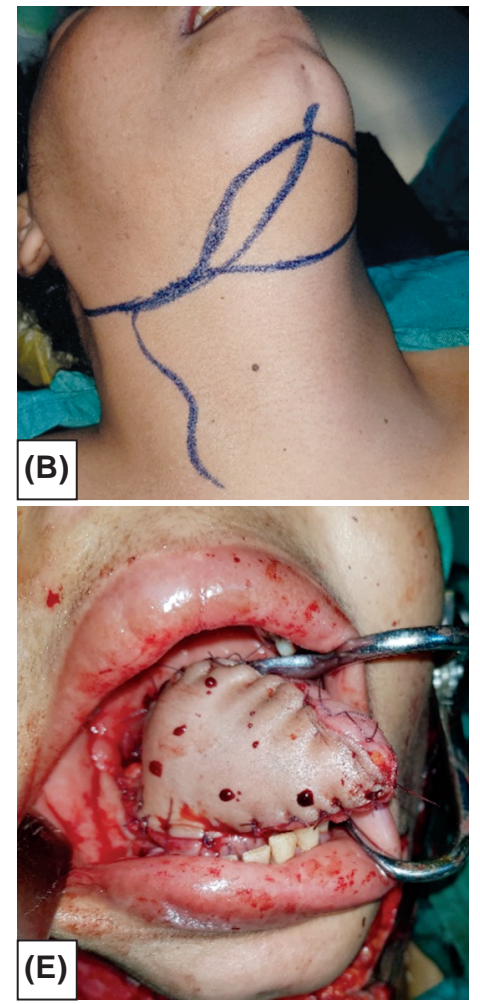
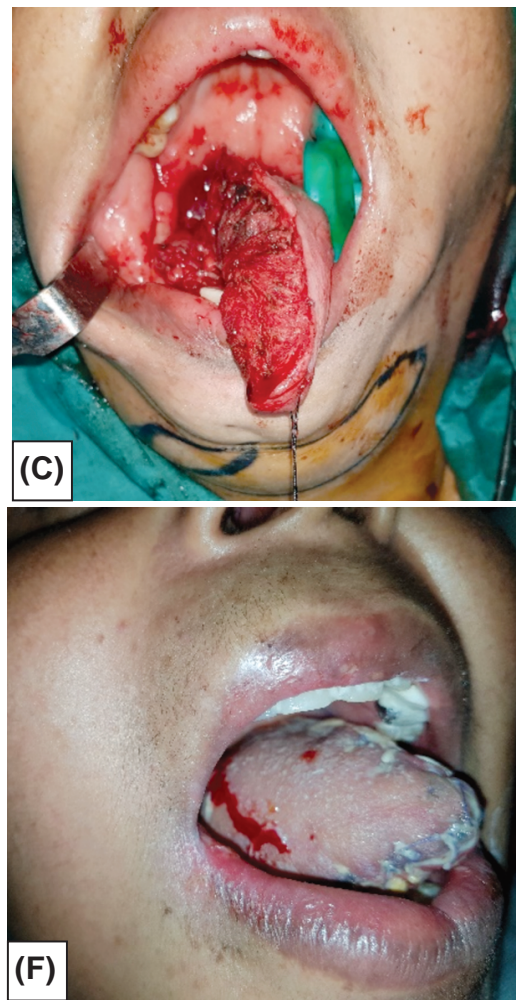

Fig. (1): A 40 years old female with carcinoma of the right side of the tongue:

(A) Preoperative view, (B) Flap design, (C) Intraoperative view showing the post-resection tongue defect, (D) Intraoperative view showing the raised submental flap, (E) Intraoperative view showing flap in position, (F) Immediate postoperative view, (G) After two months. 

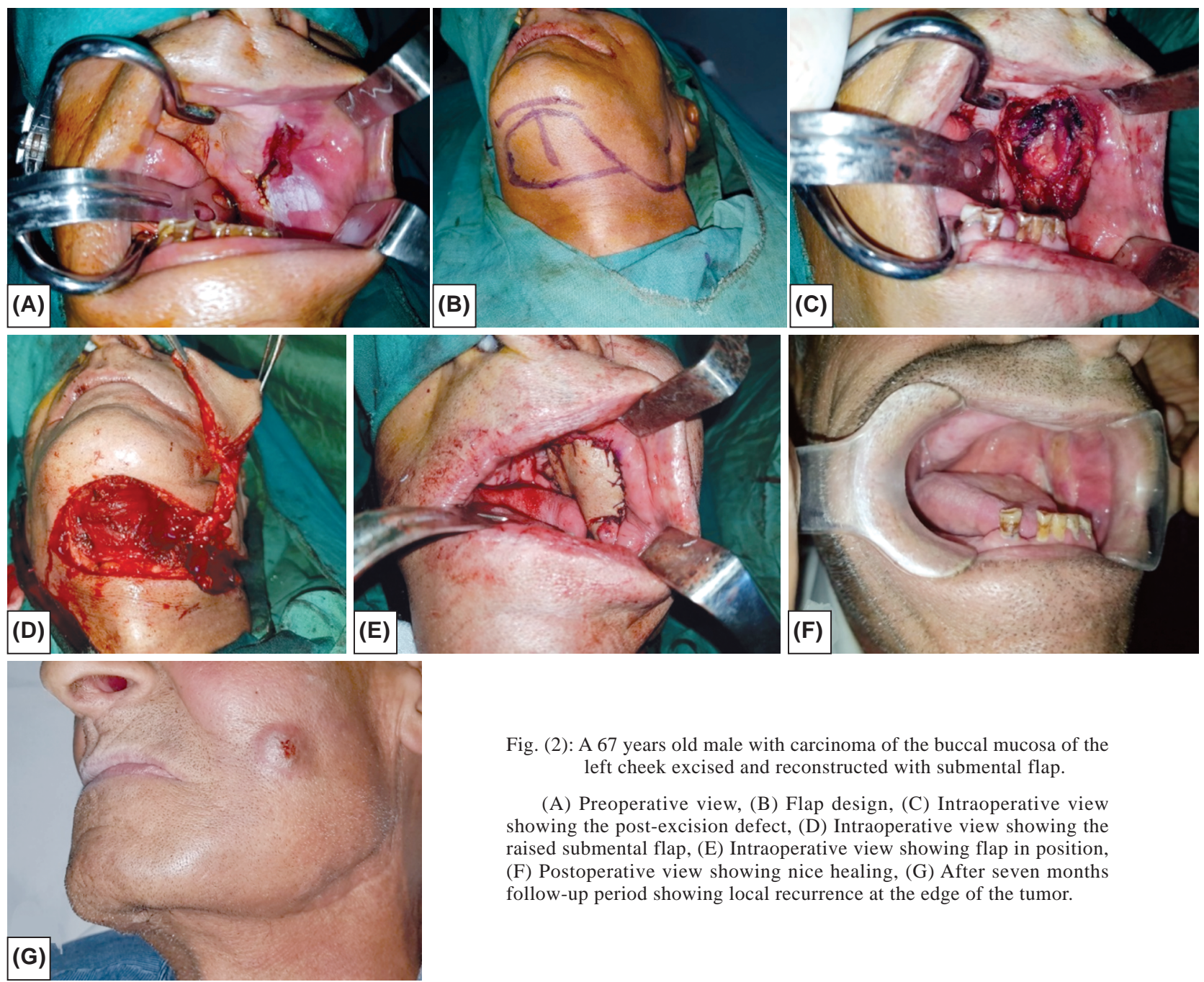

Fig. (2): A 67 years old male with carcinoma of the buccal mucosa of the left cheek excised and reconstructed with submental flap.

(A) Preoperative view, (B) Flap design, (C) Intraoperative view showing the post-excision defect, (D) Intraoperative view showing the raised submental flap, (E) Intraoperative view showing flap in position, (F) Postoperative view showing nice healing, (G) After seven months follow-up period showing local recurrence at the edge of the tumor.
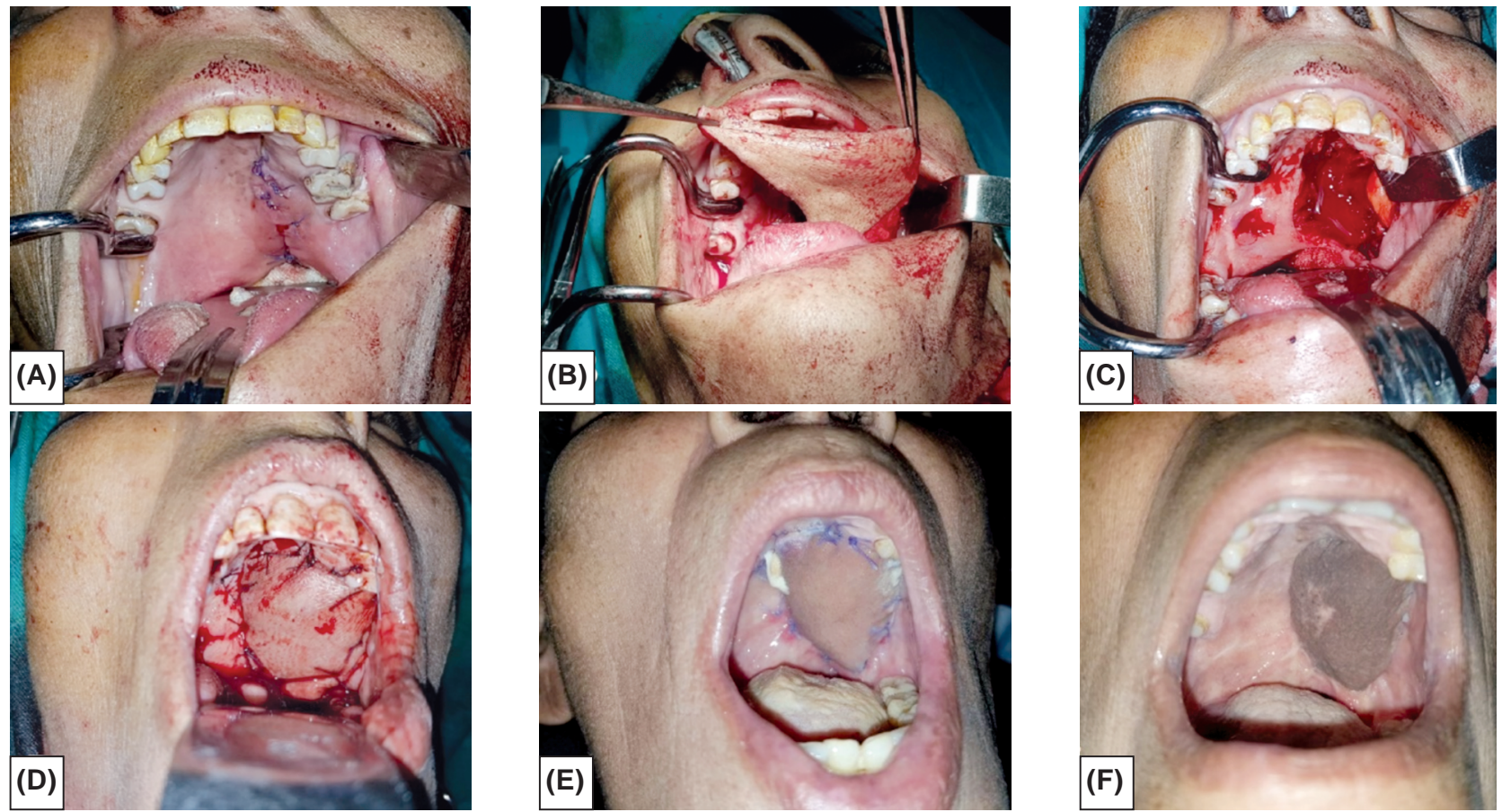

Fig. (3): A 60 years old female with carcinoma of the left maxillary alveolar margin excised and reconstructed with submental flap.

(A) Preoperative view, (B) The elevated flap, (C) Intraoperative view showing the post-excision defect, (D) Intraoperative view showing flap in position, (E) One week postoperative view, (F) After nine months. 


\section{Outcome and follow-up protocols:}

Postoperatively, all selected patients were evaluated for reconstruction efficiency, including the rate of locoregional control, the functional and cosmetic results, and the viability of the flap. The range of follow-up was 12 months. All patients received postoperative adjuvant radiotherapy to the primary site and the neck. Clinical consultations were performed every month, and regional ultrasound and or CT was performed every two months during the follow-up period. All patients received postoperative adjuvant therapy.

\section{RESULTS}

The study participants were 23 patients suffering from oral cavity carcinoma, including 15 men and eight women, with an average age of 53 years (range, 39 to 67 years). Table (1) shows the site of the primary tumor and the pathological types. Regarding the neck lymph nodes, nine patients had clinically and or radiologically negative neck before surgery, while the neck was positive clinically and or radiologically in 14 patients.

Relying on a single surgical team, all surgeries performed following the same surgical steps. In all patients, the primary tumor resected with a 1$1.5 \mathrm{~cm}$ safety margin all around, 14 patients underwent modified radical neck dissection, whereas nine patients underwent supra-omohyoid neck dissection. The dissection of the neck was completed before the flap harvesting. The flap was used to reconstruct an intra-oral soft tissue defect in 22 patients and an extra-oral soft tissue defect in one patient. All the submental flaps were transferred without being stretched, and the donor sites were closed primarily.

The harvesting of the flap took an average time of 25-40 minutes. Two flaps were partially lost, but they were managed conservatively by local debridement, and the reconstruction was not affected. Of the medially rotated flap, orocutaneous fistula developed in 2 patients, which closed spontaneously with conservative measures. Wound infection occurred in 3 patients (the two patients with fistula and another one), which healed conservatively. The donor site healed smoothly with a pleasant scar in all patients except the three patients who developed wound infection. Postoperative hospital stay ranged from 6 to 14 days. There is no indication, in any case, for admission to the ICU, especially for causes related to the flap. Postoperative functions, including swallowing, speaking, and jaw movements, were all good in all patients. There was a persistence of hair growth in the flap, and it was bothering in male patients. However, there was some improvement after radiotherapy. The marginal mandibular nerve was preserved in all cases. Postoperative irradiation does not affect flap survival; only the usual irradiation complications are encountered as dryness and scar contracture.

Regarding cervical lymph nodes, 14 patients $(61 \%)$ had ipsilateral cervical lymph node metastasis, and nine patients (39\%) were free of cervical lymph node metastasis.

Table (2) and Fig. (2G) show that five patients $(21.7 \%)$ experienced locoregional tumor relapse during follow-up. In the $\mathrm{pN}+$ group, four of 14 (28.5\%) had local-neck tumor recurrence; of these, one was a local recurrence, and three were neck recurrences. In the $\mathrm{pN} 0$ group, one of 9 (11.11\%) patients had local tumor recurrence, and there was no neck tumor recurrence (Chart 1).

All neck recurrences (4 cases) occurred in patients with tongue carcinomas (N2 two patients, $\mathrm{N} 3$ one patient, and N0 one patient), while the only case with local recurrence occurred in the patient with left cheek buccal mucosa carcinoma (N2). This patient was subjected to re-excision and reconstruction by microvascular radial forearm free flap. The other four patients who developed neck recurrences were subjected to salvage surgical resections and adjuvant chemoradiotherapy.

Table (1): Primary tumor sites and pathological types.

\begin{tabular}{ll}
\hline Variable & Number \\
\hline Primary tumor site: & \\
Tongue & 13 \\
buccal mucosa & 5 \\
Maxillary alveolar margin & 4 \\
Chin & 1 \\
Pathological types: & 17 \\
SSC & 3 \\
ACC & 2 \\
AC & 1 \\
BCC : Basal cell carcinoma. & \\
ACC : Adenoid cystic carcinoma. & \\
\hline : Adenocarcinoma. & \\
\hline
\end{tabular}


Table (2): Demographic details of patients with complications.

\begin{tabular}{|c|c|c|c|c|c|c|}
\hline \multirow{2}{*}{ Patients } & \multirow{2}{*}{ 1ry site } & \multirow{2}{*}{ TNM staging } & \multirow{2}{*}{ Pathology } & \multirow{2}{*}{ Neck dissection } & \multicolumn{2}{|c|}{ Complications } \\
\hline & & & & & Recurrence & Others \\
\hline 2 & Tongue & $\mathrm{T} 2 \mathrm{~N} 2 \mathrm{M} 0$ & SCC & MRND & Neck Recurrence & \\
\hline 4 & Tongue & $\mathrm{T} 2 \mathrm{~N} 2 \mathrm{M} 0$ & $\mathrm{AC}$ & MRND & & $\begin{array}{l}\text { - Fistula } \\
\text { - Partial flap loss } \\
\text { - Wound infection }\end{array}$ \\
\hline 5 & Buccal mucosa & T1N2M0 & $\mathrm{AC}$ & MRND & Local recurrence & \\
\hline 6 & Tongue & $\mathrm{T} 2 \mathrm{~N} 2 \mathrm{M} 0$ & SCC & MRND & & Partial flap loss \\
\hline 8 & Tongue & T2N0M0 & SCC & MRND & Neck recurrence & \\
\hline 9 & $\begin{array}{l}\text { Maxillary Alveolar } \\
\text { margin }\end{array}$ & $\mathrm{T} 2 \mathrm{~N} 0 \mathrm{MO}$ & $\mathrm{ACC}$ & SOHND & & Wound infection \\
\hline 11 & Tongue & $\mathrm{T} 2 \mathrm{~N} 1 \mathrm{M} 0$ & $\mathrm{SCC}$ & MRND & & $\begin{array}{l}\text { - Fistula } \\
\text { - Wound infection }\end{array}$ \\
\hline 13 & Tongue & $\mathrm{T} 2 \mathrm{~N} 2 \mathrm{M} 0$ & SCC & MRND & Neck Recurrence & \\
\hline 15 & Tongue & $\mathrm{T} 2 \mathrm{~N} 3 \mathrm{M} 0$ & $\mathrm{ACC}$ & MRND BILATERAL & Neck recurrence & \\
\hline
\end{tabular}

SCC : Squamous cell carcinoma. ACC : Adenoid cystic carcinoma. AC : Adenocarcinoma.

MRND : Modified radical neck dissection. SOHND: Supra-omohyoid neck dissection.

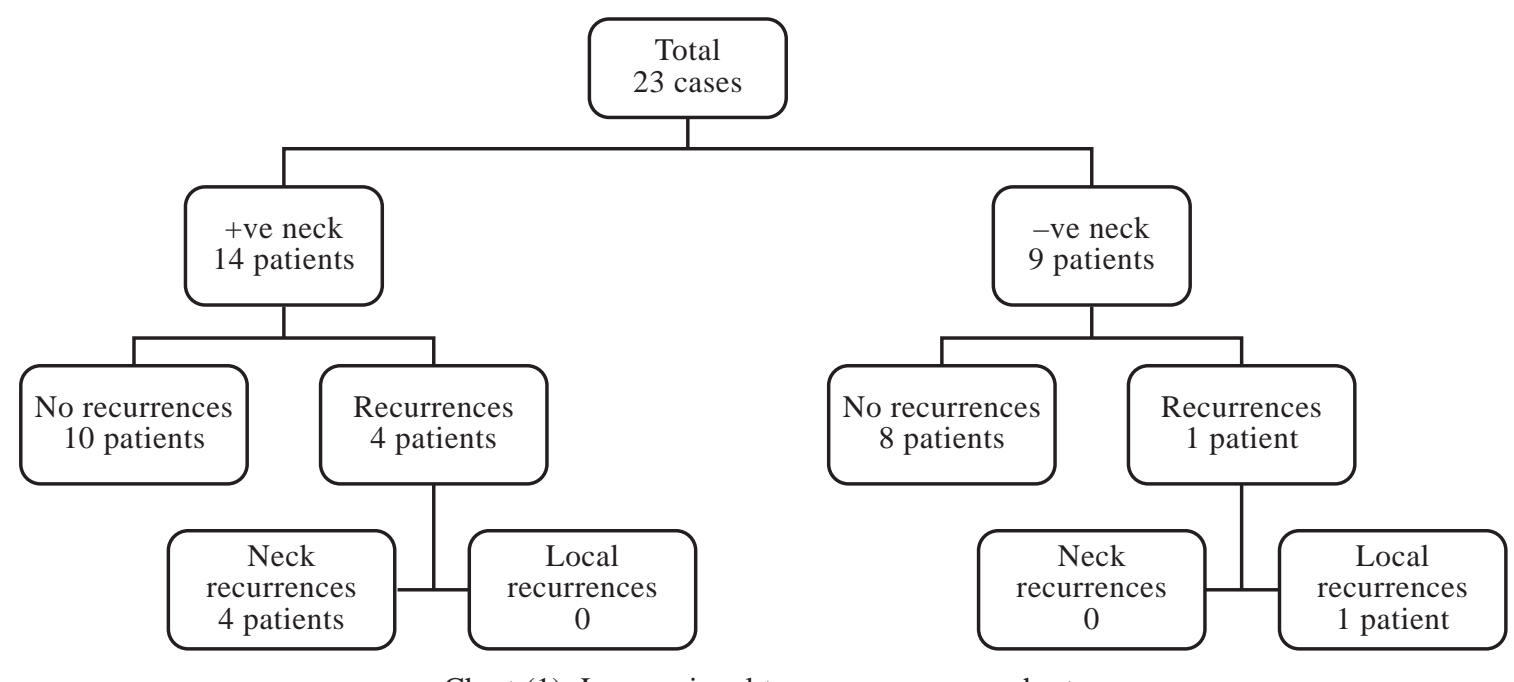

Chart (1): Locoregional tumors recurrence chart.

\section{DISCUSSION}

The main goal of treating orofacial carcinomas is to maximize the cure chance while minimizing the treatment's morbidity. With or without neck dissection, resection of the primary tumor, with at least $1-1.5 \mathrm{~cm}$, is necessary to maximize the chance of tumor clearance [3]. As a result, it is essential to maintain the reliability of reconstructing the defect, and the process of reconstruction should encourage normal functions without weakening the oncological safety [6].

There are numerous reconstructive techniques to achieve these goals, including free flaps, splitthickness skin grafts, and locoregional flaps. One of these techniques is the submental flap. With its dependent vascular supply, submental artery, a branch of the facial artery, and full arch of rotation, it is possible to employ the submental flap for reconstructing various orofacial defects [3]. However, there is no oncological safety agreement on the indication of SIF in a $\mathrm{pN}+$ setting.

Flap harvesting is more straight forward and requires no specialized microvascular flaps skills with a relatively shorter time than other flap techniques. In this study, the needed time ranged from 25 to 40 minutes, whereas radial forearm free flap harvesting required from 65 to 74 minutes, and the pectoralis major flap required from 60 to 70 minutes [7]. The submental flap does not require a second stage for dividing the pedicle as in pectoralis major or deltopectoral flaps [8]. Bree et al., [7] indicated that in 6/40, admission to ICU was revealed for radial forearm free flap. But there no indication of admission to ICU in the current study. The total management costs are less than other reconstruction 
techniques, including radial forearm free flap and pectoralis major flap; this may be due to short operative time, less hospital stay, and finally, it is a procedure of a single stage.

In this study, the anterior belly of the digastric muscle and a part of the myelohyoid muscle were included in all flaps. A partial loss was revealed in 2 flaps only, and this is consistent with others, who indicated that the flap's viability could be improved if such muscle was included [8].

The donor site's wound lies in the way of the wound of neck dissection; thus, there is no second site of wound compared to other flaps [3]. The potential risk of marginal mandibular nerve injury with submental flap harvesting ranges from $0 \%$ to 17\% [9]. Marginal mandibular nerve palsy did not occur in this study. In the study of Taghinia et al., preoperative radiotherapy was the most consistent finding in those who suffered flap loss [10]. In the current study, postoperative radiation therapy did not affect flap survival (none of the patients received preoperative radiotherapy).

This flap's concerning point is the oncological safety and potential risk of occult disease transfer to the recipient site. Studies reviewing patients undergoing submental flap reconstruction in the setting of apparent regional neck lymph node metastasis $(\mathrm{N}+)$ are few. Reconstruction in $\mathrm{N}+$ cases is even more controversial than in an N0 setting [11].

We have adopted the practice of complete lymph node dissection first before harvesting the flap; this agrees with Amin et al., who addressed that such an approach may reduce recurrence [3].

Many researchers well address the oncological safety of the submental flap in patients with the -ve neck. Kramer et al., [8] revealed the oncological safety of submental flap by comparing SIF with free radial forearm flaps in patients with $\mathrm{N} 0$ oral squamous cell carcinomas. Chang et al., [12] stated that: Based on level 3 evidence (retrospective casecontrol studies) and level 4 evidence (case series): in cases with -ve neck, it is oncologically safe to use the submental flap to reconstruct orofacial defects after oral cancer resection. There were nine patients with the -ve neck in the present study, with only one regional recurrence with no nodal recurrences.

On the other hand, there is no oncological safety agreement on the submental flap's indication in a $\mathrm{N}+$ setting [13]. In this study, there were 14 patients with a positive neck. Nodal recurrence represented
$17 \%$ of total cases and $28.5 \%$ of positive node cases. These results compared with Paydarfar and Pate's findings in their study, including 27 patients with oral cancer reconstructed by submental flap and including cases with a positive neck. Local recurrence was $26 \%$, while regional recurrence was $7.4 \%$ [13]. The higher incidence of neck recurrences than local recurrences may be attributed to the fact that the close vicinity of submental vessels to the level I group of the neck lymph nodes may jeopardize neck dissection in a trial to save the submental vessels [14].

Another critical factor that influences local tumor recurrence is the T stage. Mizrachi et al., [14] demonstrated that about $15 \%$ of patients with $\mathrm{N} 0$ oral carcinomas developed neck failure. In this study, the neck lymph node failure rate of N0 was $11.11 \%$, which was lower than that of Mizrachi's study, indicating the submental flap harvesting did not increase the risk of neck tumor recurrence in patients with T1-2 oral carcinomas. The results are in accordance with previous studies $(0-12 \%$ neck recurrence rate) [15].

Another critical factor that may influence local tumor recurrence is the biological nature of the tumor. Chow et al., addressed that cancer recurrences were more likely related to the tumors' violent nature than the flap's oncologic harm [16]. In our patients, recurrences were developed in 5 patients $(21.7 \%)$ within the follow-up period; 4 were associated with tongue primaries.

Another disadvantage of the submental flap is the growth of hair in male patients. However, after complete mucosalization over time in most patients, some improvement occurred, and after radiotherapy, that may burn the hair follicles.

This study attempted to answer the oncological safety related to reconstructing the $\mathrm{N}+$ oral carcinomas with submental flap. However, this study has limitations, including nonrandomization, small sample size, and relatively short follow-up.

\section{Conclusion:}

the submental flap is useful, easily harvested, valid, and locally available for reconstructing the orofacial surgical defects after the tumor resection, with good functional and aesthetic results. However, it is necessary to use the submental flap with adequate care in patients with a positive neck. The surgeon should never hesitate to abandon the submental flap and shift to another reconstructive option if this showed to be oncologically necessary. Carful neck dissection with postoperative radiation 
improves regional control rates in patients with +ve neck lymph nodes, provided that there is no extracapsular spread, while postoperative chemoradiation improves regional control in patients with an extracapsular spread.

\section{Conflict of interest:}

The author declares no conflict of interest.

\section{Acknowledgments:}

None.

\section{REFERENCES}

1- Jemal A., Siegel R., Ward E., et al.: Cancer statistics. CA Cancer J. Clin., 56: 106-30, 2006.

2- Sebastian P., Thomas S., Varghese B.T., et al.: The submental island flap for reconstruction of intraoral defects in oral cancer patients. Oral Oncol., 44: 1014-1018, 2008.

3- Amin A.A., Sakkary M.A., Khalil A.A., et al.: The submental flap for oral cavity reconstruction: Extended indications and technical refinements. Head Neck Oncol., 3: 1-7, 2011.

4- Martin D., Pascal J.F., Baudet J., et al.: The submental island flap: a new donor site. Anatomy and clinical applications as a free or pedicled flap. Plast. Reconstr. Surg., 92: 867-873, 1993.

5- Faisal M., Adeel M., Riaz S., et al.: The submental island flap in head and neck cancer. Ann. Maxillofac. Surg., 8: 287-291, 2018.

6- Vural E. and Suen J.Y.: The submental island flap in head and neck reconstruction. Head Neck, 22: 572-578, 2000.

7- De Bree R., Reith R., Quak J.J., et al.: Free radial forearm flap versus pectoralis major myocutaneous flap reconstruction of oral and oropharyngeal defects: A cost analysis. Clin. Otolaryngol., 32: 275-282, 2007.
8- Kramer F.J., Böhrnsen F., Moser N., et al.: The submental island flap for the treatment of intraoral tumor-related defects: No effect on recurrence rates. Oral Oncol., 51: 668-673, 2015.

9- Parmar P.S. and Goldstein D.P.: The submental island flap in head and neck reconstruction. Curr. Opin. Otolaryngol. Head Neck Surg., 17: 263-266, 2009.

10- Taghinia A.H., Movassaghi K., Wang A.X., et al.: Reconstruction of the Upper Aerodigestive Tract with the Submental Artery Flap. Plast Reconstr. Surg., 123: 562-570, 2009.

11- Paydarfar J.A. and Patel U.A.: Submental island pedicled flap vs radial forearm free flap for oral reconstruction: Comparison of outcomes. Arch. Otolaryngol. Neck Surg., 137: 82-87, 2011.

12- Chang B.A., Asarkar A.A. and Nathan C.A.O.: What is the oncologic safety of using the submental flap to reconstruct oral cavity cancer defects? Laryngoscope, 129: 2443-2444, 2019.

13- Wang J., Tan Y., Shen Y., et al.: Oncological safety of submental island flap for reconstruction of pathologically node-negative and node-positive T1-2 oral squamous cell carcinoma-related defects: A retrospective study and comparison of outcomes. Oral Oncol., 102: 104507, 2020.

14- Mizrachi A., Migliacci J.C., Montero P.H., et al.: Neck recurrence in clinically node-negative oral cancer: $27-$ year experience at a single institution. Oral Oncol., 78: 94-101, 2018.

15- Howard B.E., Nagel T.H., Donald C.B., et al.: Oncologic safety of the submental flap for reconstruction in oral cavity malignancies. In: Otolaryngology - Head and Neck Surgery (United States). Mosby Inc., pp. 558-562, 2014.

16- Chow T-L., Chan TT-F., Chow T-K., et al.: Reconstruction with submental flap for aggressive orofacial cancer. Plast. Reconstr. Surg., 120: 431-436, 2007. 